\title{
Sildenafil Use in the Treatment of Bronchopulmonary Dysplasia-Associated Pulmonary Hypertension: A Case Series
}

\author{
Amna Qasim, MD ${ }^{1}$ Soham Dasgupta, MD ${ }^{1}$ Ashraf M. Aly, MD, PhD ${ }^{2}$ Sunil K. Jain, MD ${ }^{3}$ \\ ${ }^{1}$ Department of Pediatrics, University of Texas Medical Branch, \\ Galveston, Texas \\ 2 Division of Pediatric Cardiology, University of Texas Medical Branch, \\ Galveston, Texas \\ ${ }^{3}$ Division of Neonatology, University of Texas Medical Branch, \\ Galveston, Texas \\ Am J Perinatol Rep 2018;8:e219-e222. \\ Address for correspondence Sunil K. Jain, MD, Division of \\ Neonatology, University of Texas Medical Branch, 301 University \\ Blvd., Galveston, TX 77555 (e-mail: skjain@utmb.edu).
}

Abstract
Keywords
- bronchopulmonary
dysplasia
- pulmonary
hypertension
- pulmonary
vasodilator
- NTproBNP

Objective This article studies the role of sildenafil in reducing myocardial stress (measured by serial $\mathrm{N}$-terminal pro b-type natriuretic peptide [NTproBNP] levels) secondary to bronchopulmonary dysplasia-associated pulmonary hypertension (BPD-PH).

Study Design This is a case series of three extremely low birth weight infants with severe BPD at 36 weeks' postmenstrual age. All infants had very elevated NTproBNP (>2,000 ng/dL) levels and echocardiographic evidence of BPD-PH. Sildenafil was started and infants were followed up every 2 weeks clinically along with NTproBNP levels and echocardiograms.

Results After 4 weeks of sildenafil treatment, NTproBNP levels decreased significantly in all infants, echocardiograms showed significant improvement in one infant, and respiratory severity score improved significantly in one infant. All infants tolerated sildenafil.

Conclusion Sildenafil reduced NTproBNP levels in all infants with BPD-PH but the echocardiographic findings and respiratory scores did not improve consistently. We speculate that this may be due to a delay in diagnosis and initiation of therapy after irreversible pulmonary changes have set in.
Over the last decade, survival of extremely premature infants (PIs) has improved significantly, inadvertently leading to an increased incidence of bronchopulmonary dysplasia (BPD) and BPD-pulmonary hypertension (PH)., ${ }^{1,2}$ This leads to increased morbidity and mortality in this patient population. $^{3-5}$ A joint report from the American Heart Association and the American Thoracic Society published guidelines for the management of $\mathrm{PH}$ in infants and children. ${ }^{6}$ However, specific issues like the feasibility of performing cardiac catheterizations in sick preterm infants were not addressed in detail. Recently, the Pediatric Pulmonary Hypertension Network ${ }^{7}$ presented guidelines to manage BPD-PH in preterm infants. These guidelines included baseline and serial echocardiograms (ECHOs) to determine the severity of BPD-PH along with serial Nterminal pro b-type natriuretic peptide (NTproBNP) levels to monitor disease progress.

In infants with sustained BPD-PH, phosphodiesterase 5 (PDE5) inhibitors like sildenafil may reduce pulmonary vascular resistance (PVR). Sildenafil is a pulmonary vasodilator that increases intracellular cyclic guanosine monophosphate (cGMP) levels and is U.S. Food and Drug Administration (FDA) approved for the treatment of PH in adults. ${ }^{7,8}$ However, there is limited data available to support its use in preterm infants with BPD-PH. We present a series of three PIs with BPD-PH (based on serial NTproBNP levels and ECHO evidence of $\mathrm{PH}$ ) in whom sildenafil was used as a pulmonary vascular dilatator to treat BPD-PH. received

October 11, 2017

accepted after revision

April 18, 2018
DOI https://doi.org/

10.1055/s-0038-1673343. ISSN 2157-6998.
Copyright $\odot 2018$ by Thieme Medical Publishers, Inc., 333 Seventh Avenue, New York, NY 10001, USA. Tel: +1(212) 584-4662.
License terms

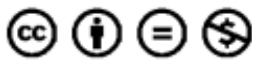


Table 1 Baseline characteristics

\begin{tabular}{|l|l|l|l|l|l|l|}
\hline Cases & GA $(\mathbf{w k} \pm \mathbf{d})$ & BW $(\mathbf{g})$ & Sex & PDA & RDS & Days of hospitalization \\
\hline 1 & $27 \pm 2$ & 490, SGA & Male & None & Surfactant (1) & 293 \\
\hline 2 & $26 \pm 2$ & 465, SGA & Male & Small, resolved spontaneously & Surfactant (3) & 237 \\
\hline 3 & $24 \pm 2$ & 675, AGA & Male & Small, resolved spontaneously & Surfactant (1) & 221 \\
\hline
\end{tabular}

Abbreviations: AGA, appropriate for gestational age; BW, birth weight; GA, gestational age; PDA, patent ductus arteriosus; RDS, respiratory distress syndrome; SGA, small for gestational age.

\section{Case Series}

Three preterm infants with severe BPD (36 weeks' postmenstrual age [PMA]) were included ( - Table $\mathbf{1}$ ) after Institutional Review Board (IRB) approval and waiver of informed consents. All infants had worsening respiratory status, increasing oxygen requirements, and significantly high NTproBNP levels $(7,585 \pm 7,079 \mathrm{ng} / \mathrm{dL})$. All infants had ECHO evidence of $\mathrm{PH}$ (right ventricular hypertrophy, flattening of the interventricular septum, pulmonary artery pressure [PAP] $>30 \mathrm{~mm} \mathrm{Hg}$ estimated by tricuspid jet velocity). ${ }^{9}$ Other comorbidities (aspiration, gastroesophageal reflux disease, structural airway disease, pulmonary arterial/venous stenosis, cardiac dysfunction) were ruled out before making the presumptive diagnosis of BPD-PH. Sildenafil was started orally and the dose was gradually increased up to $8 \mathrm{mg} / \mathrm{kg} /$ day divided four times daily. Serial NTproBNP levels were measured at 2 and 4 weeks after starting sildenafil. Respiratory severity scores (RSSs) (Mean airway pressure $\times$ Fraction of inspired oxygen $\left[\mathrm{FiO}_{2}\right]$ ) were calculated to determine respiratory support since these infants

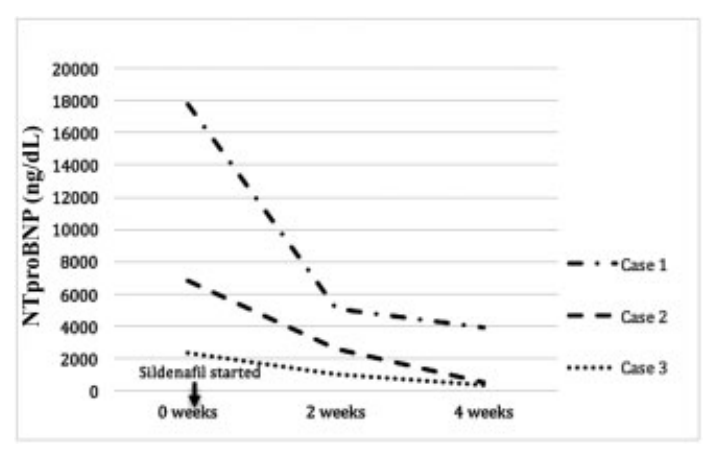

Fig. $1 \mathrm{~N}$-terminal pro b-type natriuretic peptide (NTproBNP) levels after starting sildenafil therapy. lacked arterial lines for measuring oxygenation indices. There was a significant reduction in NTproBNP levels in all the infants (-Fig. 1). RSS decreased significantly (a drop of $>20 \%{ }^{10}$ ) in 1 out of the 3 infants (62\% drop in Case 1 after 4 weeks of therapy), while the other 2 infants required an escalation in respiratory support ( - Table $\mathbf{2}$ ). ECHO was repeated at 4 weeks after starting sildenafil and showed improvement in two out of the three cases, with significant improvement ( $>20 \%$ drop in PAP) only in Case 1 (- Table 2). No significant adverse effects of sildenafil were noted.

\section{Discussion}

In our case series, we have shown that the use of NTproBNP in conjunction with ECHO was a good screening tool for BPD$\mathrm{PH}$. All infants had significantly high NTproBNP levels and ECHO changes of PH. The highest NTproBNP levels were seen in Case 1 and Case 2, likely associated with the small for gestational age status of these infants. Inhaled nitric oxide is not used by our group for the treatment of BPD-PH since the minimal benefits (7\% reduction in composite outcome of death or $\mathrm{BPD}^{11}$ ) do not outweigh the need for intubation and the high costs/resources involved.

The use of sildenafil dropped NTproBNP levels significantly in all patients and two infants (Case 1 and 3) had improved ECHO changes of $\mathrm{PH}$. However, improvement in Case 3 was $<20 \%$ and does not meet clinical significance as determined by other studies. ${ }^{10}$ RSS improved significantly in one infant while the other two required increasing respiratory support.

\section{Sildenafil in BPD-PH}

Sildenafil is a PDE5 inhibitor that mediates vasodilation by increasing intracellular cGMP levels. It also minimizes pulmonary arterial vasoreactivity and may prevent structural

Table 2 Clinical status before and 4 weeks after sildenafil therapy

\begin{tabular}{|l|l|l|l|l|l|}
\hline Cases & PCA (wk) & \multicolumn{2}{l|}{$\begin{array}{l}\text { Respiratory support/Respiratory } \\
\text { severity score (RSS) }\end{array}$} & \multicolumn{2}{l|}{ Echocardiogram } \\
\cline { 3 - 6 } & & Before & 4 wk after & Before & 4k after \\
\hline 1 & 36 & $\mathrm{HFOV} / 14$ & $\mathrm{CMV} / 5.3$ & RVH, PAP $35 \mathrm{~mm} \mathrm{Hg}$ & No RVH, No TRJV \\
\hline 2 & 37 & $\mathrm{CPAP} / 2.4$ & $\mathrm{CPAP} / 3.0$ & IVS flattening & IVS flattening, RA and RV dilatation \\
\hline 3 & 40 & $\begin{array}{l}\mathrm{HFNC} \\
\left(2 \mathrm{LPM}, 25 \% \mathrm{FiO}_{2}\right)\end{array}$ & $\begin{array}{l}\mathrm{HFNC} \\
\left(6 \mathrm{LPM}, 21 \% \mathrm{FiO}_{2}\right)\end{array}$ & $\begin{array}{l}\text { Mild RVH, } \\
\text { PAP } 30 \mathrm{~mm} \mathrm{Hg}\end{array}$ & PAP $26 \mathrm{~mm} \mathrm{Hg,} \mathrm{No} \mathrm{RVH}$ \\
\hline
\end{tabular}

Abbreviations: CMV, continuous mechanical ventilation; CPAP, continuous positive airways pressure; $\mathrm{FiO}_{2}$, fraction of inspired oxygen; HFNC, high flow nasal cannula; HFOV, high frequency oscillation ventilation; IVS, interventricular septum; LPM, liter per minute; PAP, estimated pulmonary artery pressure; PCA, gestational age at start of sildenafil therapy; RA, right atrium; RV, right ventricular; RVH, right ventricular hypertrophy. 
remodeling in the pulmonary vessels. ${ }^{12} \mathrm{~A}$ pilot randomized controlled study suggested that oral sildenafil may reverse neonatal persistent PH. ${ }^{13}$ In 2013, FDA cautioned against the use of sildenafil in children due to concerns of increased mortality observed in children receiving high doses. ${ }^{14}$ However, several studies have shown the efficacy of off-label use of sildenafil in the treatment of BPD-PH without any significant adverse effects. Two case series of sildenafil treatment of infants with BPD-PH demonstrated improved pulmonary pressures without adverse effects. ${ }^{6}$ Mourani et al conducted a retrospective review of 25 patients $<2$ years of age that were treated with sildenafil for BPD-PH and reported hemodynamic improvement in $88 \%$ of the patients after a median treatment duration of 40 days. ${ }^{15}$ One patient had recurrent erections necessitating cessation of therapy, no other adverse effects were noted. Wardle et al demonstrated that sildenafil may improve survival in children with BPD-PH if therapy is continued until resolution of $\mathrm{PH} .{ }^{8}$ Tan et al reviewed charts of 22 infants that were treated with sildenafil for BPD-PH and showed a significant improvement in echocardiographic markers of $\mathrm{PH}$ at 27.5 days (interquartile range, 24,31 ) in two-thirds of infants as well as a significant decrease in the mean $\mathrm{FiO}_{2} \cdot{ }^{16} \mathrm{~A}$ retrospective study by Nyp et al reported similar findings to our study where the use of sildenafil in 21 preterm infants was associated with a reduction in estimated right ventricular peak systolic pressures but the majority of the infants showed no improvement in gaseous exchange after 48 hours of therapy. ${ }^{10}$ The authors attributed the lack of improvement to ventilation/perfusion (V/Q) mismatch secondary to alterations in pulmonary blood flow and inhibition of hypoxic vasoconstriction. ${ }^{10}$ Trottier-Boucher et al conducted a retrospective review of 23 PIs treated with sildenafil for BPD-PH and found significant echocardiographic responses in $71 \%$ of infants but clinical responses (defined by $20 \%$ decrease in respiratory support score of $\mathrm{FiO}_{2}$ ) were only seen in 35\% of the infants. ${ }^{17}$ We speculate that the late initiation of pulmonary vasodilator therapy (after irreversible pulmonary vascular changes had set in) may play a role in the lack of improvement in respiratory status in some infants.

\section{Screening for BPD-PH}

A prospective cohort study on 60 very PIs demonstrated that elevated BNP (B-type natriuretic peptide) levels at 36 weeks' PMA were significantly associated with the severity of BPD. ${ }^{18}$ A retrospective study evaluated the role of BNP and ECHO in screening for BPD-PH. BNP levels were measured within 10 days of the ECHO. They found that the median BNP levels were significantly higher in infants with BPD-PH $(p<0.001)$ and further a BNP value of $117 \mathrm{pg} / \mathrm{mL}$ had $93.8 \%$ sensitivity and $100 \%$ specificity for detecting BPD-PH. ${ }^{19}$ A recent study enrolled PIs ( $<27$ weeks' gestational age [GA] and/or with a birth weight of $\leq 750 \mathrm{~g}$ ), who met the criteria for BPD at 36 weeks' PMA. A screening ECHO at 36 to 38 weeks' PMA and plasma NTproBNP levels were significantly elevated in the BPD-PH group and the authors concluded that NTproBNP levels $>1,000 \mathrm{pg} / \mathrm{mL}$ may be a cost-effective screening biochemical marker for BPD-PH in PI. ${ }^{20}$ We have also demon- strated that NTproBNP levels correlate well with ECHO evidence of BPD-PH (unpublished data-submitted to Journal of Perinatology). We measured NTproBNP levels in PIs $(<30$ weeks' GA and $<1,500 \mathrm{~g}$ birth weight) at 28, 32, and 36 weeks' PMA. ECHO was also performed within 12 hours of obtaining NTproBNP levels to look for evidence of $\mathrm{PH}$. Preliminary results have shown that neonates with ECHO evidence of PH had significantly elevated levels of NTproBNP $(\geq 2,300 \mathrm{pg} / \mathrm{mL})$ at 28,32 , and 36 weeks' PMA $(p<0.05)$. In our case series, we also found NTproBNP levels and ECHO were useful tools to screen for BPD-PH.

Impaired angiogenesis during alveolar development leading to decreased vascular surface area, pulmonary arterial remodeling, and increased PVR are the proposed pathophysiological mechanisms involved in BPD-PH. ${ }^{21}$ Prolonged exposure to increased right ventricular pressure leads to myocardial remodeling and pulmonary arterial fibrotic changes that are usually irreversible. Those infants may also be exposed to prolonged mechanical ventilation for progressive lung disease. Since these changes may begin long before 36 weeks' PMA, infants in our case series might have already developed irreversible pulmonary changes. This would explain the lack of improvement in respiratory status with sildenafil treatment. A prospective cohort study showed that the ratio of endostatin (an antiangiogenic factor) to angiopoiten-1 (a pro-angiogenic factor) was significantly higher on day 1 (median, 31.8 vs. 11.3) and day 7 (median, 62.1 vs. 19) in the BPD-PH group versus the no BPD group. ${ }^{22}$ An increased endostatin to angiopoiten-1 ratio may reflect impaired angiogenesis and may be used for early biochemical screening of BPD-PH. Genetic and epigenetic biomarkers are also being studied as possible biomarkers for early screening of BPD-PH. ${ }^{23}$

\section{Are We Screening Early Enough?}

We speculate that the initiation of sildenafil at $\geq 36$ weeks' PMA in our case series improved pulmonary arterial pressures and reduced stress on the right heart (indirectly reflected by the significant drop in NTproBNP levels). However, this did not translate into consistent clinical improvement, as seen in other studies mentioned above, ${ }^{10,17}$ likely due to irreversible changes in the pulmonary vasculature. Earlier screening and treatment of BPD-PH may be helpful to prevent these irreversible changes and improve short- and long-term clinical outcomes. Further studies of the natural history, screening biomarkers, and impact of therapeutic interventions like PDE5 inhibitors in BPD-PH are required. Early screening for BPD-PH with biomarkers like serial NTproBNP along with ECHO may be helpful in the diagnosis and management before irreversible changes occur.

\section{Conclusion}

The use of sildenafil in infants with BPD-PH improved rightsided pressures inconsistently but led to a consistent decline in markers of cardiac stress. We speculate that the lack of improvement in respiratory status in some infants may be due to irreversible pulmonary changes secondary to delay in 
diagnosis and initiation of pulmonary vasodilator therapy. Developing an early screening protocol for BPD-PH in PIs with significant respiratory disease (escalation of support/ increased oxygen requirement) may allow early initiation of therapeutic intervention with better outcomes. Multicenter studies are required to identify infants that need to be screened, develop screening protocols, and to determine the best timing of therapeutic intervention.

\section{Conflict of Interest}

None.

\section{References}

1 Ehrenkranz RA, Walsh MC, Vohr BR, et al; National Institutes of Child Health and Human Development Neonatal Research Network. Validation of the National Institutes of Health consensus definition of bronchopulmonary dysplasia. Pediatrics 2005;116 (06):1353-1360

2 Kinsella JP, Greenough A, Abman SH. Bronchopulmonary dysplasia. Lancet 2006;367(9520):1421-1431

$3 \mathrm{Kim}$ DH, Kim HS, Choi CW, Kim EK, Kim BI, Choi JH. Risk factors for pulmonary artery hypertension in preterm infants with moderate or severe bronchopulmonary dysplasia. Neonatology 2012;101 (01):40-46

4 Mourani PM, Abman SH. Pulmonary vascular disease in bronchopulmonary dysplasia: pulmonary hypertension and beyond. Curr Opin Pediatr 2013;25(03):329-337

5 Mirza H, Ziegler J, Ford S, Padbury J, Tucker R, Laptook A. Pulmonary hypertension in preterm infants: prevalence and association with bronchopulmonary dysplasia. J Pediatr 2014; 165(05):909-14.e1

6 Abman SH, Hansmann G, Archer SL, et al; American Heart Association Council on Cardiopulmonary, Critical Care, Perioperative and Resuscitation; Council on Clinical Cardiology; Council on Cardiovascular Disease in the Young; Council on Cardiovascular Radiology and Intervention; Council on Cardiovascular Surgery and Anesthesia; and the American Thoracic Society. Pediatric pulmonary hypertension: guidelines from the American Heart Association and American Thoracic Society. Circulation 2015;132(21):2037-2099

7 Krishnan U, Feinstein JA, Adatia I, et al; Pediatric Pulmonary Hypertension Network (PPHNet). Evaluation and management of pulmonary hypertension in children with bronchopulmonary dysplasia. J Pediatr 2017;188:24-34.e1

8 Wardle AJ, Wardle R, Luyt K, Tulloh R. The utility of sildenafil in pulmonary hypertension: a focus on bronchopulmonary dysplasia. Arch Dis Child 2013;98(08):613-617

9 Nagiub M, Lee S, Guglani L. Echocardiographic assessment of pulmonary hypertension in infants with bronchopulmonary dysplasia: systematic review of literature and a proposed algorithm for assessment. Echocardiography 2015;32(05):819-833

10 Nyp M, Sandritter T, Poppinga N, Simon C, Truog WE. Sildenafil citrate, bronchopulmonary dysplasia and disordered pulmonary gas exchange: any benefits? J Perinatol 2012;32(01):64-69

11 Donohue PK, Gilmore MM, Cristofalo E, et al. Inhaled nitric oxide in preterm infants: a systematic review. Pediatrics 2011;127(02): e414-e422

12 Baker CD, Abman SH, Mourani PM. Pulmonary hypertension in preterm infants with bronchopulmonary dysplasia. Pediatr Allergy Immunol Pulmonol 2014;27(01):8-16

13 Baquero H, Soliz A, Neira F, Venegas ME, Sola A. Oral sildenafil in infants with persistent pulmonary hypertension of the newborn: a pilot randomized blinded study. Pediatrics 2006;117 (04):1077-1083

14 Rubin LJ, Badesch DB, Fleming TR, et al; SUPER-2 Study Group. Longterm treatment with sildenafil citrate in pulmonary arterial hypertension: the SUPER-2 study. Chest 2011;140(05):1274-1283

15 Mourani PM, Sontag MK, Ivy DD, Abman SH. Effects of long-term sildenafil treatment for pulmonary hypertension in infants with chronic lung disease. J Pediatr 2009;154(03):379-384, 384.e1-384.e2

16 Tan K, Krishnamurthy MB, O’Heney JL, Paul E, Sehgal A. Sildenafil therapy in bronchopulmonary dysplasia-associated pulmonary hypertension: a retrospective study of efficacy and safety. Eur J Pediatr 2015;174(08):1109-1115

17 Trottier-Boucher MN, Lapointe A, Malo J, et al. Sildenafil for the treatment of pulmonary arterial hypertension in infants with bronchopulmonary dysplasia. Pediatr Cardiol 2015;36(06): $1255-1260$

18 Kalra VK, Aggarwal S, Arora P, Natarajan G. B-type natriuretic peptide levels in preterm neonates with bronchopulmonary dysplasia: a marker of severity? Pediatr Pulmonol 2014;49(11): 1106-1111 [Erratum in Pediatr Pulmonol 2015;50(5):423]

19 Cuna A, Kandasamy J, Sims B. B-type natriuretic peptide and mortality in extremely low birth weight infants with pulmonary hypertension: a retrospective cohort analysis. BMC Pediatr 2014; 14:68

20 Montgomery AM, Bazzy-Asaad A, Asnes JD, et al. Biochemical Screening for Pulmonary Hypertension in Preterm Infants with Bronchopulmonary Dysplasia. Neonatology 2016;109(03):190-194

21 Berkelhamer SK, Mestan KK, Steinhorn RH. Pulmonary hypertension in bronchopulmonary dysplasia. Semin Perinatol 2013;37 (02):124-131

$22 \mathrm{Kim} \mathrm{DH}, \mathrm{Kim}$ HS. Serial changes of serum endostatin and angiopoietin-1 levels in preterm infants with severe bronchopulmonary dysplasia and subsequent pulmonary artery hypertension. Neonatology 2014;106(01):55-61

23 Bui CB, Pang MA, Sehgal A, et al. Pulmonary hypertension associated with bronchopulmonary dysplasia in preterm infants. J Reprod Immunol 2017;124:21-29 